\title{
Container-Based Clinical Solutions for Portable and Reproducible Image Analysis
}

\author{
Jordan Matelsky $^{1}$ - Gregory Kiar ${ }^{2} \cdot$ Erik Johnson $^{1} \cdot$ Corban Rivera $^{1} \cdot$ Michael Toma ${ }^{1} \cdot$ William Gray-Roncal ${ }^{1}$ \\ Published online: 8 May 2018 \\ (C) The Author(s) 2018
}

\begin{abstract}
Medical imaging analysis depends on the reproducibility of complex computation. Linux containers enable the abstraction, installation, and configuration of environments so that software can be both distributed in self-contained images and used repeatably by tool consumers. While several initiatives in neuroimaging have adopted approaches for creating and sharing more reliable scientific methods and findings, Linux containers are not yet mainstream in clinical settings. We explore related technologies and their efficacy in this setting, highlight important shortcomings, demonstrate a simple use-case, and endorse the use of Linux containers for medical image analysis.
\end{abstract}

Keywords Containers $\cdot$ Reproducibility $\cdot$ Docker $\cdot$ Singularity $\cdot$ Medical-imaging

\section{Introduction}

The evolution of complex imaging methods, coupled with reductions in the cost of data storage, have led to increasingly large data in the clinical imaging community. In parallel, computational methods to analyze these images have grown more sophisticated and nuanced. While these new techniques represent exciting steps forward in stateof-the-art medical image processing, they also expose the field to new operational vulnerabilities: researchers must take special care to precisely emulate the computational environment and configuration of an algorithm developer, or risk the possibility of incorrect results. As neural networks and other complex analyses grow in popularity, ensuring that analyses are reproducible and repeatable has taken on new importance.

Even well-designed and highly compatible software can incur issues when researchers' computers do not perfectly match software developers' operating system, configuration, or installed libraries. Resulting findings may therefore fail to truly enable experiment reproducibility.

Jordan Matelsky

jordan.matelsky@jhuapl.edu

1 Applied Physics Laboratory, Johns Hopkins University, 11100 Johns Hopkins Road, Laurel, MD 20723-6099, USA

2 McGill University, Montreal, QC, Canada
Invented in 2008, Linux containers provide a way to "freeze" an environment in a simple package, enabling serialization and redeployment $[1,2]$. However, Linux containers in their basic form remain relatively esoteric and difficult to use for the majority of non-expert users. For this reason, many software solutions exist to simplify the process of tool or environment containerization. Containerization enables the distribution of software bundled alongside its required packages and libraries, increasing the portability of software and the ability to reproduce scientific analyses. Docker and Singularity are two emerging platforms which provide user-friendly avenues for containerization and serve distinct communities based upon differences in core features such as data security and accessibility [3, 4].

\section{Background}

Researchers have adopted a variety of strategies in order to homogenize compute platforms to enable reproducible software-based analyses. Some tools, such as Python virtual environments and Conda, modify the local environment, but cannot provide cross-platform reproducibility or extend beyond specific language barriers [5]. Virtual machines (VMs) are one form of portable cross-platform compute environments that enable highly reproducible compute environments regardless of host operating system or environment [6]. Some cloud compute providers such as 
Amazon Web Services (AWS) offer their own versions of these-the most popular being Amazon Machine Images (AMIs) — which are commonly used and distributed, but only available on specific vendors' computing resources [7]. Unfortunately, virtual machines are costly in terms of resource usage. VMs must contain a full operating system which leads to large amounts of overhead, even for simple analytical pipelines [8]. Because VMs are fully virtualized computers, they must be "booted up," which dramatically increases the amount of time required in order to perform an analysis. Furthermore, VMs most often require that resources such as RAM or hard-drive space are fully devoted and allocated prior to the first boot up. This means that it is increasingly difficult to run multiple virtual machines on the same host machine, even when the VMs are idle (Fig. 1a).

Containers are another tool commonly tapped for the purposes of resource abstraction. Like virtual machines, containers enable rapid deployment of tools across various infrastructures, and they shift the burden of installation to tool developers rather than users. These environments can be deployed consistently across computational infrastructures and are useful for debugging and understanding tools. Unlike virtual machines, containers do not require resource
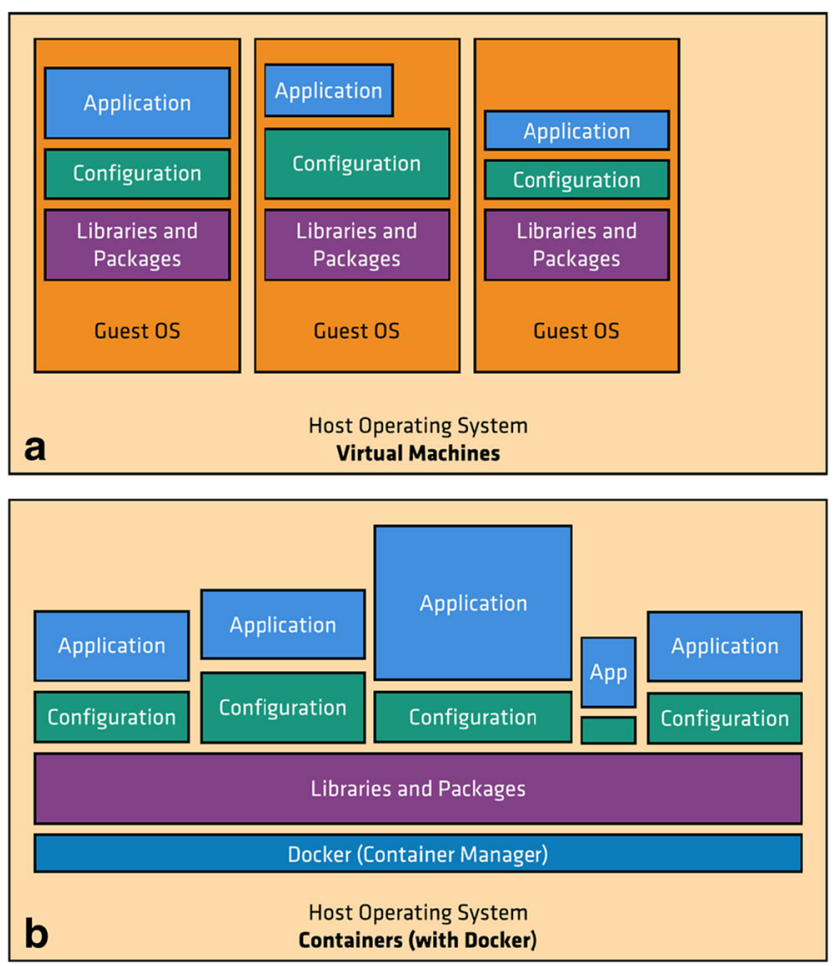

Fig. 1 a Virtual machines each require their own guest operating system (OS), libraries, and configuration. The VMs pictured above have pre-allocated sizes and use hard drive space and RAM even when idle. b In contrast, containers do not require a guest operating system, can share libraries, and only the resources needed for a particular analysis allocation at creation time: Rather, resources are shared in real time with the host system (Fig. 1b).

Containerized software has seen wide adoption in the software development community, for both commercial (AWS [9], Google Gloud [10], Azure [11], etc.) and academic platforms (SIC [12], CBRAIN [13], Virtual Imaging Platform [14]). These groups have adopted containers as a way to repeatably install and deploy tools. Adoption efforts by neuroimaging groups have helped to bring containerization into the forefront of the medical imaging analysis consciousness. In particular, container-based neuroimaging pipelines such as MRI analysis serve as an efficient vehicle for sharing code and analyses between development environments and higher-performance production workloads.

\section{Container Managers}

Both Singularity and Docker provide self-contained environments which enable command-line or graphical execution of tools or services. Each of these systems leverages the host system's kernel for performing tasks, meaning that while the encapsulated tools are identical, the underlying execution may differ slightly across computational infrastructures.

\section{Docker}

Docker is the most widely used container management system because it is installable on all major operating systems and its configuration language is mature, simple, and well documented [15]. This makes Docker a highly accessible solution for developing and wrapping tools. Docker's major limitation is that of security: The software inside the container can be granted elevated permissions, in which case malicious software could access or manipulate the host system. This is often not an issue on personal workstations or commercial clouds, as the host systems are small, isolated from one another, and contain little persistent data, and so we recommend Docker for these settings [15] (Table 1).

\section{Singularity}

Singularity's major contribution addresses Docker's security shortcomings. Singularity restricts permissions within a container to those of the user launching the task. Singularity also enables HIPAA-compliant access-logging and differential privacy [16]. For this reason, Singularity is the platform of choice for many high-performance or high-security compute environments.

Singularity has seen less adoption due to its more recent introduction and inability to run on Windows or OSX systems. 
It therefore has a smaller support community and base of documentation than Docker, but enables the importing and conversion of most Docker containers, which reduces the need for developers to become intimately familiar with both frameworks.

\section{Use-Cases for Containerization}

One common pitfall when deploying software in a controlled environment is that of operating system versioning. Systems are often "frozen" to older versions of an operating system in order to either maintain compatibility with mission-critical software or pause at a known stable or secure version of a supported OS. This becomes a problem when new research products require more modern libraries than the underlying OS natively supports.

This problem is solved by installing a container manager, such as Docker, on the older platform. Barring extenuating concerns, including inability to install such software on the older platform or numerical precision fragility (discussed below in Risks and Limitations), containerized software will deliver the same results on out-of-date host operating systems as it would on modern ones, without incurring the additional labor and cost of upgrading resources.

\section{Workflow Optimization}

A common convention is to run atomic steps of an analysis in separate containers. That is, rather than installing all software in one large container, each container holds only a single step. For an imaging pipeline, this might mean that a

Table 1 A comparison of the basic features of Docker and Singularity

\begin{tabular}{lll}
\hline Feature & Docker & Singularity \\
\hline Secure & $\times$ & $\checkmark$ \\
Scalable & $\checkmark$ & $\checkmark$ \\
Cross-compatible & $\times$ & $\checkmark$ \\
Supports all major OSes & $\checkmark$ & $\times$ \\
Accessible documentation & $\checkmark$ & $\times$
\end{tabular}

Each of these platforms provides a powerful lightweight solution to reliable, portable computing. Aside from this, Docker benefits from a large user community, rich documentation, and the ability to be deployed easily on all major operating systems (including Windows), whereas Singularity is less mature in these areas. The differentiating strength of Singularity lies in its ability to be deployed securely across shared high-performance computing infrastructures, preserving user access restrictions, whereas Docker is not suitable for these applications. Singularity is also capable of converting Docker images, lending itself to the popular use-case of being a deployment engine for containers developed locally through Docker color correction step lives in the first container, an alignment step lives in the second, and an image processing step such as lesion detection lives in the third.

Between steps, byproducts are stored in volumes, or mountable directories that can be shared with the host OS so that files persist after the container is destroyed [17]. This enables complex error handling or retry behavior: if the first step of an analysis fails, no other containers need to be run; if the second step fails, the results of the first step do not need to be recalculated. Furthermore, multiple algorithms with different requirements can be integrated by building Docker containers with different installation dependencies.

This stepwise paradigm is so common that there are many existing pipelining systems that fulfill just this need [18-21].

These tools, in conjunction with containers, enable detailed logging and rich capturing of execution outputs and progress. This provides reliable work histories for reproducible analysis and debugging.

\section{Integrating Docker into a Clinical Workflow}

\section{The Project}

In a hypothetical workflow, a scientist may want to normalize the contrast of an MRI scan in order to increase contrast. A developer on the team writes a Python script called normalize_scan.py that takes three arguments from the command-line:

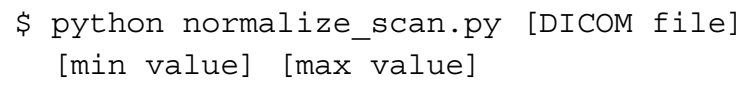

and outputs the same file with the suffix -normalized, with the data remapped to the minimum/maximum values provided.

These scientists work on a shared host machine, and the software dependencies for this tool may conflict with those used by other labs.

\section{Writing a Dockerfile}

If this tool were run on a new system, the scientists would get the Python Module Not Found error telling them that their environment was not properly configured with the libraries required to run their script. Luckily, the software developer provided a requirements . txt which lists all required Python libraries.

In this simple case, the scientists can simply read the imports from the Python file and install them, but this method is less tractable for applications which depend upon code written in multiple languages, or those developed by multiple disparate teams. 
The team decides to execute their code inside a Docker container in order to prevent version conflicts and to enable workflow reproducibility.

Leveraging prior work by other open-source developers, the scientists can use an existing container that has certain libraries pre-installed. For the purpose of illustration, we'll use pydicom/dicom [22]. (In clinical environments, it may be wiser to build the container from scratch in order to have full control over the execution environment and eliminate unneeded dependencies.)

To indicate this to the image-building software packaged with Docker, the Dockerfile will begin with the line:

\section{FROM pydicom/dicom}

It is considered best practice to include the name of the maintainer of this Dockerfile, in case future users have questions:

LABEL maintainer="Your Name

<your@email.com>"

The next step is to copy the Python script to the image:

$\mathrm{ADD}$./normalize_scan.py /src/

normalize_scan.py

....as well as the requirements.txt file. The following line executes pip, the Python package manager, in order to install the libraries listed in this file.

$\mathrm{ADD}$./requirements.txt /src/requirements.txt RUN pip install -r/src/requirements.txt

Finally, the Dockerfile indicates to Docker what command it should run when the container starts by providing an "entrypoint." In this example, all of the scans will be remapped to the [0..100] range. One could allow for userdefined inputs using environment variables, as we do in code provided at https://github.com/jdi-matelsky-et-al-2018/.

ENTRYPOINT python3/src/normalize_scan.py /infile.dcm/mnt/vout/ 0100

This code makes the assumption that our scan exists at the path /infile.dcm. This is a safe assumption, as we'll discuss in Section 2.

\section{Building the Image}

Next, the Docker image can be built. The image may take some time to build while it downloads and installs packages, but this only needs to be done when changes are made to the contents of the image: Afterward, Docker containers can be launched quickly based on this image template.

To build the container and tag it with the name of remap-dicom, the scientists will run the following command:

docker build -t username/dicom-remap .

where the "username" field above may correspond to the scientist's account on Docker Hub, a sharing platform for Docker images [23]. This enables the image builder to (optionally) "push" the image and download it on other machines or, if shared publicly, enable others to use it.

This command will retrieve the latest version of the pydicom/dicom image from Docker Hub and will then run the commands from the above Dockerfile in order, caching the results of each step so that successive builds are faster and more efficient.

\section{Building a Singularity Image}

If the scientists wish for their tool to be available for others who are using platforms which require Singularity rather than Docker, they can easily leverage the above work in order to create a Singularity image hosted instead on Singularity Hub. There are two common approaches for this: The first is through the docker2singularity utility [24], and the second is by creating a Singularity file. The former method is an open-source tool which walks the user through the conversion of Docker images to Singularity images. The latter is a much shorter equivalent of the Dockerfile created above but it relies on pushing the Docker image to Docker Hub. If the image is accessible online, this file can contain just the two lines below:

Bootstrap: docker From: username/dicom-remap

\section{Running Docker Containers}

The final step is running the container. To do this, the scientist navigates into the directory with the image file they wish to normalize, called myfile.dem and runs the following command:

mkdir. /out docker run -v $\$(\mathrm{pwd}) /$

myfile.dcm:/infile.dcm -v $\$(p w d) /$

out:/mnt/vout dicom-remap

The $-\mathrm{v}$ syntax indicates a Docker volume: This is a mapping from the host's filesystem to the private Docker filesystem. Because actions inside the container do not affect the host environment, the scientist must deliberately allow the container to make changes to only specific files. 
Two volumes are mounted by the command above. The first is the target file, myfile.dcm, which is mounted to the /infile.dcm location (referenced earlier). Simply, this allows the container to access this under the alias of /infile.dcm.

The second mounted volume is the output directory. An empty directory on the host file system is mounted, \$ (pwd)/out, to the directory /mnt/vout/ in the container. When a file is placed in the container's /mnt/vout/ directory, it will appear in the host machine's \$ (pwd) / out/ directory.

By mounting these locations rather than a full working directory, data access is restricted within the container. Deliberate and careful use of volumes improves container security.

\section{Risks and Limitations}

While containers simplify many aspects of dependency management and analysis reproducibility, this technology requires that a user manage container installation and execution. This may provide a barrier to entry in cases where potential users lack the necessary training. Enduser container management applications are typically straightforward, and writing new recipes (i.e, Dockerfiles) may be unfamiliar to some researchers. Despite this, we observe that existing tutorials and seed containers make this straightforward for most users.

Containers may reduce the accessibility of containerbased tools when users are restricted to environments that do not support container management systems (e.g. old operating systems or platforms that dramatically restrict user permissions). This can be mitigated by system administrators aiming to provide up-to-date environments for researchers.

Because container use abstracts operating system selection and installation processes from tool users, the tool user has less control over the eventual execution environment. This can lead to incompatibilities that are not obvious to the end user. One particularly subtle example of this is the practice of pointing to the "latest" version of libraries when installing. This makes the image brittle to changes in updates to its dependency libraries. If another user builds the image after a library has updated its latest-deployed version, the resultant image will differ from the author's. Users can easily remedy this issue by explicitly specifying recommended versions of libraries or software when distributing images.

Recent research has suggested that the use of containers may obfuscate the origin of numerical instabilities running inside the container. These differences may only become apparent when deploying software across multiple operating systems using the same data and comparing the results. Several recent studies (in submission, OHBM 2018 [25]) have demonstrated significant differences in MRI brain segmentation when running the popular PreFreeSurfer pipeline [26] between different operating systems and configurations. It is suspected that these differences between operating systems are the result of underlying numerical instabilities in the underlying algorithms, and numerical libraries may be performing different approximations or handling of these exceptions.

We assert that these issues are irrelevant for the majority of users and suggest exercising caution and validating results when deploying software both when using and when not using containers.

\section{Discussion}

Because clinical and medical sciences are increasingly reliant on complex algorithms in order to derive insight from data, analysis repeatability or reproducibility should be considered a high priority (see [27] for a review of these terms and their various definitions). Many medical image analysis developers are beginning to provide their tools in containerized environments, enabling portable, repeatable analyses. Spearheading this containerization effort are Singularity and Docker, two platforms that simplify the process of developing and deploying containerbased applications. These systems hold the promise of dramatically reducing the complexity of configuring and installing medical imaging analysis tools and pipelines.

These tools have been deployed in a wide range of infrastructures, including privacy-sensitive settings such as the XSEDE super computing cluster in the USA [28], Compute Canada [29], and other commercial clouds [9]. While adoption in clinical research environments is still low, these platforms can serve as valuable tools when bridging the gap between research and clinical settings.

Acknowledgements Research reported in this publication was supported by the National Institute of Mental Health of the National Institutes of Health under Award Number R24MH114799. The content is solely the responsibility of the authors and does not necessarily represent the official views of the National Institutes of Health.

Funding Information This work was funded by the Canada First Research Excellence Fund, awarded to McGill University for the Healthy Brains for Healthy Lives initiative.

Open Access This article is distributed under the terms of the Creative Commons Attribution 4.0 International License (http:// creativecommons.org/licenses/by/4.0/), which permits unrestricted use, distribution, and reproduction in any medium, provided you give appropriate credit to the original author(s) and the source, provide a link to the Creative Commons license, and indicate if changes were made. 


\section{References}

1. Tanenbaum AS: Modern operating system. Pearson Education, Inc, 2009

2. Soltesz S, Pötzl H, Fiuczynski ME, Bavier A, Peterson L: Container-based operating system virtualization: a scalable, high-performance alternative to hypervisors. In: ACM SIGOPS Operating Systems Review, vol 41. ACM, 2007, pp 275-287

3. Docker Accessed: 2018-03-15. https://www.docker.com

4. Singularity Accessed: 2018-03-15. http://singularity.lbl.gov

5. Virtual Environment virtualenv Documentation Accessed: 201803-15. https://virtualenv.pypa.io/en/stable/

6. Clark C, Fraser K, Hand S, Hansen JG, Jul E, Limpach C, et al: Live migration of virtual machines. In: Proceedings of the 2nd Conference on Symposium on Networked Systems Design \& Implementation-Volume 2. USENIX Association, 2005, pp 273286

7. Amazon Web Services AMIs Accessed: 2018-03-15. https://docs. aws.amazon.com/AWSEC2/latest/UserGuide/AMIs.html

8. Goldberg RP: Survey of virtual machine research. Computer 7(6):34-45, 1974

9. Amazon Web Services FarGate Accessed: 2018-03-15. https:// aws.amazon.com/fargate/

10. Google Cloud Accessed: 2018-03-15. https://cloud.google.com/ compute/docs/containers/

11. Microsoft Azure Accessed: 2018-03-15. https://azure.microsoft. com/en-us/overview/containers/

12. Kiar G, Gorgolewski KJ, Kleissas D, Roncal WG, Litt B, Wandell B, et al: Science in the cloud (SIC): A use case in MRI connectomics. GigaScience 6(5):1-10, 2017. Available from: https://doi.org/10.1093/gigascience/gix013

13. Sherif T, Rioux P, Rousseau ME, Kassis N, Beck N, Adalat R, et al: CBRAIN: A web-based, distributed computing platform for collaborative neuroimaging research. Frontiers in Neuroinformatics 8:54, 2014

14. Glatard T, Lartizien C, Gibaud B, Da Silva RF, Forestier G, Cervenansky $\mathrm{F}$, et al: A virtual imaging platform for multimodality medical image simulation. IEEE Trans Med Imaging 32(1):110-118, 2013

15. Merkel D: Docker: Lightweight Linux Containers for Consistent Development and Deployment. Linux J 2014(239):76-91, 2014. Available from: http://dl.acm.org/citation.cfm?id=2600239. 2600241
16. Kurtzer GM, Sochat V, Bauer MW: Available from: https://doi. org/10.1371/journal.pone.0177459. PLOS ONE 12(5):e0177459, 2017

17. Di Tommaso P, Palumbo E, Chatzou M, Prieto P, Heuer ML, Notredame C: The impact of Docker containers on the performance of genomic pipelines. PeerJ 3:e1273, 2015. Available from: https://doi.org/10.7717/peerj.1273

18. Hindman B, Konwinski A, Zaharia M, Ghodsi A, Joseph AD, Katz $\mathrm{R}$, et al: Mesos: A Platform for Fine-grained Resource Sharing in the Data Center. In: Proceedings of the 8th USENIX Conference on Networked Systems Design and Implementation. NSDI'11. USENIX Association, Berkeley, 2011, pp 295-308. Available from: http://dl.acm.org/citation.cfm?id=1972457.1972488

19. Burns B, Grant B, Oppenheimer D, Brewer E, Wilkes J: Borg, Omega, and Kubernetes. ACM Queue 14:70-93, 2016. Available from: http://queue.acm.org/detail.cfm?id=2898444

20. Afgan E, Baker D, Van den Beek M, Blankenberg D, Bouvier D, ČEch M, et al: The Galaxy platform for accessible, reproducible and collaborative biomedical analyses: 2016 update. Nucleic Acids Res 44(W1):W3-W10, 2016

21. Awesome Pipelines: Accessed: 2018-03-15. https://github.com/ pditommaso/awesome-pipeline/

22. Mason D: SU-E-T-33: Pydicom: An Open Source DICOM Library. Med Phys 38(6Part10):3493-3493, 2011. Available from: https://doi.org/10.1118/1.3611983

23. Docker Hub: Accessed: 2018-03-15. https://hub.docker.com/

24. Docker Hub: Accessed: 2018-03-15. https://github.com/singularity ware/docker2singularity

25. Gronenschild EHBM, Habets P, Jacobs HIL, Mengelers R, Rozendaal N, van Os J, et al: The Effects of FreeSurfer Version, Workstation Type, and Macintosh Operating System Version on Anatomical Volume and Cortical Thickness Measurements. PLoS ONE 7(6):e38234, 2012. Available from: https://doi.org/10.1371/ journal.pone.0038234

26. Fischl B: Freesurfer. Neuroimage 62(2):774-781, 2012

27. Plesser HE: Reproducibility vs. Replicability: A Brief History of a Confused Terminology. Frontiers in neuroinformatics 11:76, 2018

28. Towns J, Cockerill T, Dahan M, Foster I, Gaither K, Grimshaw A, et al: XSEDE: Accelerating Scientific discovery. Computing in Science \& Engineering 16(5):62-74, 2014

29. Baldwin S: Compute Canada: Advancing Computational Research. In: Journal of Physics: Conference Series, vol 341. IOP Publishing, 2012, p 012001 